Pacific Journal of Mathematics

ON CLASS NUMBERS OF CYCLIC QUARTIC FIELDS 


\title{
ON CLASS NUMBERS OF CYCLIC QUARTIC FIELDS
}

\author{
TSUYOSHI UEHARA
}

Let $n$ be a given natural number and $F$ a quadratic field contained in a cyclic quartic field. In this paper we shall construct infinitely many imaginary cyclic quartic fields containing $F$ whose relative class numbers are divisible by $n$.

1. Introduction. Let $K$ be an imaginary abelian number field, $K^{+}$ its maximal real subfield, and let $h$ and $h^{+}$be the respective class numbers. It is known that $h^{+}$divides $h$. The quotient $h^{-}=h / h^{+}$is called the relative class number of $K$. The purpose of this paper is to give a complement of a result in our previous paper [3]. Namely we shall prove the following

THEOREM. Let $F$ be a quadratic field contained in a cyclic quartic field. Then there exist infinitely many imaginary cyclic quartic fields containing $F$ each with relative class number divisible by a given integer.

It is seen from Lemma 2 in the next section that for a square free rational integer $m$ the quadratic field generated by $m^{1 / 2}$ is contained in a cyclic quartic field if and only if $m=s^{2}+t^{2}$ for some rational integers $s, t$.

2. Lemmas. By $Z, Q$ we denote the ring of rational integers, the field of rational numbers respectively. For any number field $L$ let $C(L)$ be the ideal class group of $L$.

LeMma 1 (for instance, cf. [2], Ch. 3, Theorem 4.3). Let $K$ be an imaginary abelian number field, and $K^{+}$its maximal real subfield. Let $\phi$ be the norm mapping from $C(K)$ to $C\left(K^{+}\right)$and put $C^{-}(K)=\operatorname{Ker} \phi$. Then $\phi$ is surjective, and the relative class number of $K$ is equal to the order of $C^{-}(K)$.

LEMMA 2 ( $c f .[1])$. Let $m \neq 1$ be a square free rational integer and $a, b$ rational numbers. Put $\eta=a+b m^{1 / 2}$. Then $Q\left(\eta^{1 / 2}\right)$ is a cyclic quartic field if and only if $a^{2}-b^{2} m=c^{2} m$ for some $c$ in $Q$. 
We now take rational integers $s, t$ for which $m=s^{2}+t^{2}$ is square free and put

$$
\eta=f\left(m+t m^{1 / 2}\right), \quad \boldsymbol{\theta}=\eta^{1 / 2},
$$

$f$ being a square free rational integer. By Lemma $2, K=Q(\theta)$ is a cyclic quartic field. Let $\sigma$ be a generator of the Galois group $\operatorname{Gal}(K / Q)$. Then $\left(\theta^{\sigma}\right)^{2}=f\left(m-t m^{1 / 2}\right)$. We put $\omega=m^{1 / 2}$ if $m$ is even and $\omega=$ $\left(1+m^{1 / 2}\right) / 2$ if $m$ is odd. Note that $\theta^{\sigma^{2}}=-\theta$ and $\omega^{\sigma^{2}}=\omega$.

LEMMA 3. Let the notation be as above. If $p$ is an odd prime dividing $f$, then for any integer $\alpha$ in $K$ and any $k>0$ in $Z$ there is an integer $\beta$ in $Q\left(m^{1 / 2}\right)$ such that

$$
\alpha^{p^{k}} \equiv \beta \quad\left(\bmod p^{k}\right) .
$$

If $m^{\prime} \equiv 0(\bmod 2)$ or $f \equiv t(\bmod 2)$, the above assertion is also valid for $p=2$.

Proof. First we remark that if $\alpha^{p} \equiv \beta(\bmod p)$ is true for some $\beta$ in $Z[\omega]$ then the assertion is easily shown by induction on $k$.

Let $p$ be an odd prime dividing $f$. We can find integers $a, b, c, d$ in $Z$ such that

$$
\alpha \equiv\left(a+b \omega+c \theta+d \theta^{\sigma}\right) / p^{e}(\bmod p), \quad e \geq 0 .
$$

Since $\alpha+\alpha^{\sigma^{2}} \equiv 2(a+b \omega) / p^{e}(\bmod p)$ we have $a \equiv b \equiv 0\left(\bmod p^{e}\right)$. Hence $\pi=\left(c \theta+d \theta^{\sigma}\right) / p^{e}$ is an integer and $\alpha-\pi \equiv \beta_{1}(\bmod p)$ holds for some $\beta_{1}$ in $Z[\omega]$. Observing $c \pi-d \pi^{\sigma}=\left(c^{2}+d^{2}\right) \theta / p^{e}$ we get $c^{2}+d^{2} \equiv 0\left(\bmod p^{e}\right)$. We compute

$$
p^{2 e} \pi^{2}=\left(c^{2}+d^{2}\right) f m+\left\{\left(c^{2}-d^{2}\right) t \pm 2 c d s\right\} f m^{1 / 2} .
$$

If $e=0$ then $\pi^{2} \equiv 0(\bmod p)$. When $e>0$ we may assume $(c, p)=$ $(d, p)=1$. We derive $c t \pm d s \equiv 0\left(\bmod p^{e}\right)$. This implies that $s \equiv \pm l c$ $\left(\bmod p^{e}\right), t \equiv-l d\left(\bmod P^{e}\right)$ for some $l$ in $Z$. Hence $m \equiv l^{2}\left(c^{2}+d^{2}\right) \equiv 0$ $\left(\bmod p^{e}\right)$ and so $e=1$. Notice that $p \geq 5$ and $\pi^{4} \equiv 0(\bmod p)$ in this case. Thus $\pi^{p} \equiv 0(\bmod p)$ and $\alpha^{p} \equiv \beta_{1}^{p}(\bmod p)$ in all cases.

To verify the assertion in the case $p=2$ we put $\xi=\left(\theta+\theta^{\sigma}\right) / 2$, $\xi^{\prime}=\left(\theta-\theta^{\sigma}\right) / 2$ and suppose that for some $u, v, x, y$ in $Z, \zeta=$ $\left(u+v \omega+x \xi+y \xi^{\prime}\right) / 2$ is an integer. We shall show that $u, v, x$ and $y$ are all even. We write $4 \zeta \zeta^{\sigma^{2}} \equiv M+N \omega$ with $M, N$ in $Z$. Clearly $M \equiv N \equiv$ $0(\bmod 4)$. In the case that $m$ is even, one sees

$$
\left\{\begin{array}{l}
M=u^{2}+v^{2} m-\left(x^{2}+y^{2}\right) f m / 2 \\
N=2 u v-\left\{x y t \pm\left(x^{2}-y^{2}\right) s / 2\right\} f .
\end{array}\right.
$$


Since $s$ and $t$ are both odd and $f \neq \equiv(\bmod 4)$, we get $x \equiv y(\bmod 2)$. This implies $u \equiv 0(\bmod 2)$ and hence $N \equiv-x y t \equiv 0(\bmod 2)$. The last congruence shows $x \equiv y \equiv 0(\bmod 2)$. Thus $v$ is also even. Next let $m$ be odd. Then

$$
\left\{\begin{array}{l}
M=u^{2}+v^{2}(m-1) / 4-\left\{\left(x^{2}+y^{2}\right) m \pm\left(x^{2}-y^{2}\right) s-2 x y t\right\} f / 2 \\
N=(2 u+v) v+\left\{ \pm\left(x^{2}-y^{2}\right) s-2 x y t\right\} f .
\end{array}\right.
$$

If $f$ and $t$ are both even, we have $v \equiv 0(\bmod 2)$ and $x \equiv y(\bmod 2)$ because $(2 u+v) v \pm\left(x^{2}-y^{2}\right) f s \equiv 0(\bmod 4)$ and $s$ is odd. Hence it follows from $M \equiv 0(\bmod 4)$ that $u \equiv x \equiv y \equiv 0(\bmod 2)$. If $f$ and $t$ are both odd, since $s$ is even, we first see $v \equiv 0(\bmod 2)$. Observing $2 M \equiv$ $\left(x^{2}+y^{2}\right) f m \equiv 0(\bmod 2)$ we have $x \equiv y(\bmod 2)$. From $N \equiv-2 x y f t$ $\equiv 0(\bmod 4)$ one can derive $x \equiv y \equiv 0(\bmod 2)$. Thus $u$ is also even. The above argument shows that under the assumption $\alpha \equiv \beta_{1}+c \xi+d \xi^{\prime}$ $(\bmod 2)$ holds with $\beta_{1}$ in $Z[\omega]$ and $c, d$ in $Z$. Here $\xi_{1}=c \xi+d \xi^{\prime}$ is an integer and $\xi_{1}^{2}$ is in $Z[\omega]$. This yields that $\alpha^{2} \equiv \beta(\bmod 2)$ with $\beta$ in $Z[\omega]$. Hence the proof is complete.

3. Proof of the theorem. In the following, for any prime $p$ and any rational integer $g$, ord $g$ means the exponent of the exact power of $p$ dividing $g$. Let $m=s^{2}+t^{2}$ be a square free rational integer with $s, t>0$ in $Z$. For a given natural number $n$ we put

$$
n^{\prime}= \begin{cases}2^{3} n^{2} & \text { if } n \text { is even and } m t \text { is odd } \\ 2^{2} n^{2} & \text { if } n \text { and } m t \text { are both even } \\ n^{2} & \text { if } n \text { is odd }\end{cases}
$$

Proposition. Let the notation be as above. Take rational integers a, $b>0$ satisfying

(i) $(a, b t)=1$,

(ii) $\left(a^{2}-b^{2} t^{2} m, 2 m s\right)=1$,

(iii) ord $_{p} b=1$ for every prime $p$ dividing $n$,

(iv) $A-B m>0$,

where $A+B t m^{1 / 2}=\left(a+b t m^{1 / 2}\right)^{n^{\prime}}$ with $A, B$ in $Z$. Moreover put

$$
\eta=\left(2 B m-A+B t m^{1 / 2}\right)^{2}-\left(A+B t m^{1 / 2}\right)^{2} .
$$

Then $K=Q\left(\eta^{1 / 2}\right)$ is an imaginary cyclic quartic field, and the relative class number of $K$ is divisible by $n$ unless $K$ is the fifth cyclotomic field.

Proof. Computing $\eta=4 B(B m-A)\left(m+t m^{1 / 2}\right)$ we obtain the first assertion from Lemma 2 and (iv). We put

$$
\alpha=a+b t m^{1 / 2}, \quad \beta=2 B m-A+B t m^{1 / 2}, \quad \theta=\eta^{1 / 2} .
$$


Then $(\beta+\theta)(\beta-\theta)=\alpha^{2 n^{\prime}}$. Suppose that there is a prime ideal $P$ of $K$ dividing both the integers $\beta \pm \theta$. Let $p$ be the prime lying below $P$. Then $p$ divides $a^{2}-b^{2} t^{2} m$ because $\alpha$ is in $P$. By (ii) we have $(p, 2 m s)=1$. The congruence $a \equiv-b t m^{1 / 2}(\bmod P)$ implies $B t m^{1 / 2} \equiv-2^{n^{\prime}-1} a^{n^{\prime}}$ $(\bmod P)$. Hence from $(\mathrm{i})$ we can derive $(p, B)=1$. On the other hand $2 \alpha^{n^{\prime}}+2 \beta=4 B\left(m+t m^{1 / 2}\right)$ is contained in $P$ and hence $p$ must divide $2 B m s$. This gives a contradiction. Thus $(\beta+\theta, \beta-\theta)=1$ and $(\beta+\theta)$ $=I^{2 n^{\prime}}$ holds for some ideal $I$ of $K$. The ideal class represented by $I$ belongs to $C^{-}(K)$, which was defined in Lemma 1 , because $I I^{\tau}=(\alpha)$, where $\tau$ is the generator of the Galois group $\operatorname{Gal}\left(K / Q\left(m^{1 / 2}\right)\right)$.

Let $p$ be any prime dividing $n$. From (iii) it is easy to see $\operatorname{ord}_{p}\left(\begin{array}{l}n^{\prime} \\ l\end{array}\right) b^{2}$ $>1+\operatorname{ord}_{p} n^{\prime}$ for any odd integer $i, 3 \leq i \leq n^{\prime}$. By (i) we get $(a, p)=1$. Hence it follows that $(A, p)=1$ and $\operatorname{ord}_{p} B=1+\operatorname{ord}_{p} n^{\prime}$. We write $4 B(B m-A)=r^{2} f$, where $r, f$ are in $Z$ and $f$ is square free. Let $l=\operatorname{ord}_{p} n$. Then we obtan

$$
\operatorname{ord}_{p} r= \begin{cases}l+3 & \text { if } p=2 \text { and } m t \text { is odd, } \\ l+2 & \text { if } p=2 \text { and } m t \text { is even, } \\ l & \text { if } p>2\end{cases}
$$

Moreover $f$ is divisible by every odd prime dividing $n$, and $f \equiv t(\bmod 2)$ is valid if $n$ is even and $m$ is odd.

We now assume ord $C^{-}(K)<l$. We put $k=\operatorname{ord}_{p} 2 n^{\prime}$ and consider the ideal $J=I^{2 n^{\prime} / p^{k}}$. Then $J^{p^{l-1}}=(\zeta)$ for some integer $\zeta$ in $K$. Hence $\beta+\theta=\varepsilon \zeta^{p^{k-1+1}}$ holds, $\varepsilon$ being a unit of $K$. We know that $\varepsilon_{1}=\varepsilon / \varepsilon^{\tau}$ is a root of unity. Since $Q\left(\varepsilon_{1}\right) \subset K$, it is seen from Lemma 2 that $\varepsilon_{1}= \pm 1$ if $K$ is not equal to the fifth cyclotomic field. By means of Lemma 3 we have

$$
\zeta^{p^{k-l+1}} \equiv\left(\zeta^{\tau}\right)^{p^{k-1+1}} \equiv \xi \quad\left(\bmod p^{k-l+1}\right)
$$

for some $\xi$ in $Z[\omega]$. Thus $\beta+\theta \equiv \pm(\beta-\theta)\left(\bmod p^{k-l+1}\right)$. Since $\beta \equiv$ $-A \neq \equiv(\bmod p)$, it holds that

$$
2 \theta= \pm 2 r\left(f \eta^{\prime}\right)^{1 / 2} \equiv 0 \quad\left(\bmod p^{k-l+1}\right)
$$

with $\eta^{\prime}=m+t m^{1 / 2}$. On the other hand ord $2 r<k-l+1$. Therefore $f \eta^{\prime}$ must be divisible by $p^{2}$. But this is impossible. Hence the order of $C^{-}(K)$ is a multiple of $p^{l}$. This proves the second assertion.

Proof of Theorem. Let $K_{i}(i=1, \ldots, g)$ be a finite number of quartic fields each generated by $\left(f_{l} \eta^{\prime}\right)^{1 / 2}$ with $f_{l}$ in $Z$ and $\eta^{\prime}=m+t m^{1 / 2}$. To prove the theorem it suffices to find an imaginary cyclic quartic field different from any $K_{i}$ such that $h^{-} \equiv 0(\bmod n)$. Take a prime $q$ not dividing $10 f_{1} \cdots f_{g} m n$ and choose a positive rational integer $b$ satisfying 
the condition (iii) and $\operatorname{ord}_{q} b=1$. The condition (iv) is equivalent to the inequality

$$
\left(m^{1 / 2}+t\right)\left(a-b t m^{1 / 2}\right)^{n^{\prime}}>\left(m^{1 / 2}-t\right)\left(a+b t m^{1 / 2}\right)^{n^{\prime}} .
$$

By simple computation we see that if $t>s$ and $a>3 b n^{\prime} t m^{1 / 2}$ then (iv) is valid. Hence we can find an integer $a>0$ in $Z$ satisfying (i), (ii) and (iv). Let $K$ be the field generated by $\left(f^{\prime} \eta\right)^{1 / 2}$ over $Q$ with $f^{\prime}=4 B(B m-A)$, where $A, B$ are defined as in Proposition. It is clear that ord $f^{\prime}=1$ and $K$ is not equal to the fifth cyclotomic field. Further $K \neq K_{i}$ for any $i$, $1 \leq i \leq g$. Indeed, if $K=K_{i}$ for some $i$, then $\left(f^{\prime} / f_{i}\right)^{1 / 2}$ is contained in the quadratic field $Q\left(m^{1 / 2}\right)$. This contradicts the choice of $q$. Hence $K$ is a desired field, and the proof is complete.

\section{REFERENCES}

[1] H. Edgar and B. Peterson, Some contributions to the theory of cyclic quartic extensions of the rationals, J. Number Theory, 12 (1980), 77-83.

[2] S. Lang, Cyclotomic Fields, Springer-Verlag, New York, 1978.

[3] T. Uehara, On class numbers of imaginary quadratic and quartic fields, Arch. Math. (Basel), 41 (1983), 256-260.

Received November 15, 1984.

SAGA UNIVERSITY

SAGA, JAPAN 



\section{PACIFIC JOURNAL OF MATHEMATICS EDITORS}

\author{
V. S. VARADARAJAN (Managing Editor) \\ University of California \\ Los Angeles, CA 90024 \\ Hebert Clemens \\ University of Utah \\ Salt Lake City, UT 84112 \\ Charles R. DePrima \\ California Institute of Technology \\ Pasadena, CA 91125
}

R. FINN

Stanford University

Stanford, CA 94305

HermanN FlaschKa

University of Arizona

Tucson, AZ 85721

RAMESH A. GANGOLlI

University of Washington

Seattle, WA 98195

ROBION KIRBY

University of California

Berkeley, CA 94720

ASSOCIATE EDITORS
R. ARENS
E. F. BECKENBACH
B. H. NEUMANN
F. WOLF
K. YoshidA (1906-1982)

C. C. Moore

University of California

Berkeley, CA 94720

H. SAMELSON

Stanford University

Stanford, CA 94305

HAROLD STARK

University of California, San Diego

La Jolla, CA 92093

\section{SUPPORTING INSTITUTIONS}

UNIVERSITY OF ARIZONA

UNIVERSITY OF BRITISH COLUMBIA

CALIFORNIA INSTITUTE OF TECHNOLOGY

UNIVERSITY OF CALIFORNIA

MONTANA STATE UNIVERSITY

UNIVERSITY OF NEVADA, RENO

NEW MEXICO STATE UNIVERSITY

OREGON STATE UNIVERSITY
UNIVERSITY OF OREGON

UNIVERSITY OF SOUTHERN CALIFORNIA

STANFORD UNIVERSITY

UNIVERSITY OF HAWAII

UNIVERSITY OF TOKYO

UNIVERSITY OF UTAH

WASHINGTON STATE UNIVERSITY

UNIVERSITY OF WASHINGTON 


\section{Pacific Journal of Mathematics}

Vol. 122, No. $1 \quad$ January, 1986

Michael James Cambern, Near isometries of Bochner $L^{1}$ and $L^{\infty}$ spaces ....1 Kun Soo Chang, Gerald William Johnson and David Lee Skoug, The

Feynman integral of quadratic potentials depending on two time

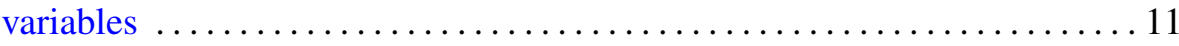

Robert Coleman, One-dimensional algebraic formal groups $\ldots \ldots \ldots \ldots \ldots 35$

Alberto Collino, The Abel-Jacobi isomorphism for the cubic fivefold .......43

N. J. Dev and S. S. Khare, Finite group action and vanishing of $N_{*}^{G}[F] \ldots 57$

Harold George Diamond and Jeffrey D. Vaaler, Estimates for partial sums

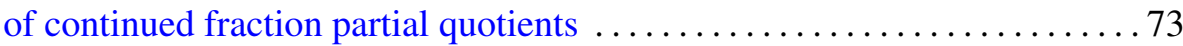

Kenneth R. Goodearl, Patch-continuity of normalized ranks of modules

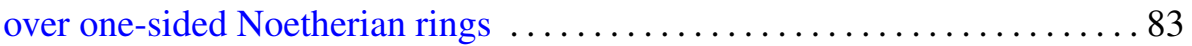

Dean Robert Hickerson and Sherman K. Stein, Abelian groups and

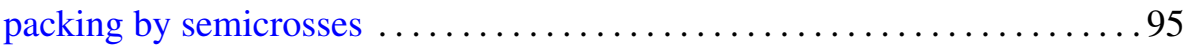

Karsten Johnsen and Harmut Laue, Fitting structures $\ldots \ldots \ldots \ldots \ldots 11$

Darren Long, Discs in compression bodies . ................... 129

Joseph B. Miles, On the growth of meromorphic functions with radially distributed zeros and poles ........................... 147

Walter Volodymyr Petryshyn, Solvability of various boundary value problems for the equation $x^{\prime \prime}=f\left(t, x, x^{\prime}, x^{\prime \prime}\right)-y \ldots \ldots \ldots \ldots \ldots . \ldots 169$

Elżbieta Pol, The Baire-category method in some compact extension problems

Masami Sakai, A new class of isocompact spaces and related results 211

Thomas Richard Shemanske, Representations of ternary quadratic forms and the class number of imaginary quadratic fields ..

Tsuyoshi Uehara, On class numbers of cyclic quartic fields 